\title{
Segregation distortion and the evolution of sex-determining mechanisms
}

\author{
M Kozielska ${ }^{1,2}$, FJ Weissing ${ }^{2}$, LW Beukeboom ${ }^{1}$ and I Pen ${ }^{2}$ \\ ${ }^{1}$ Evolutionary Genetics, University of Groningen, Haren, The Netherlands and ${ }^{2}$ Theoretical Biology, University of Groningen, Haren, \\ The Netherlands
}

\begin{abstract}
Segregation distorters are alleles that distort normal segregation in their own favour. Sex chromosomal distorters lead to biased sex ratios, and the presence of such distorters, therefore, may induce selection for a change in the mechanism of sex determination. The evolutionary dynamics of distorter-induced changes in sex determination has only been studied in some specific systems. Here, we present a generic model for this process. We consider three scenarios: a driving $X$ chromosome, a driving $Y$ chromosome and $a$ driving autosome with a male-determining factor. We investigate how the invasion prospects of a new sexdetermining factor are affected by the strength of distortion and the fitness effect of the distorting allele. Our models show that in many cases, segregation distortion does create
\end{abstract}

selection pressure, allowing novel sex-determining alleles to spread. When distortion leads to female-biased sex ratios, a new masculinizing gene can invade, leading to a new male heterogametic system. When distortion leads to male-biased sex ratios, a feminizing factor can invade and cause a switch to female heterogamety. In many cases, the distorterinduced change in the sex-determining system eventually leads to loss of the distorter from the population. Hence, the presence of sex chromosomal distorters will often only be transient, and the distorters may remain unnoticed. The role of segregation distortion in the evolution of sex determination may, therefore, be underestimated.

Heredity (2010) 104, 100-112; doi:10.1038/hdy.2009.104; published online 12 August 2009

Keywords: segregation distortion; sex determination; meiotic drive; sex chromosome

\section{Introduction}

Most chromosomes follow 'fair' Mendelian segregation, resulting in each of the homologues being present in (approximately) 50\% of the gametes. However, some genetic elements are recovered in more than half of the functional gametes of heterozygous individuals showing so-called segregation distortion or meiotic drive. Segregation distortion occurs in a number of taxa, ranging from fungi to plants and animals (for reviews see, for example, Jaenike, 2001; Burt and Trivers, 2006) with marked similarity between taxa (Taylor and Ingvarsson, 2003).

Segregation distortion is advantageous at the gene level, as distorter alleles have a transmission advantage and their frequency in the population will increase. Many distorters in nature show almost complete distortion when unsuppressed (the distorter allele is present in more than $90 \%$ of functional gametes). However, considerable variation exists between populations and different distorters, and an effective distortion can range from just above 0.5 to almost 1 (for example, see Sturtevant and Dobzhansky, 1936; Hickey and Craig, 1966; Gileva, 1987; Carvalho de et al., 1989; Jaenike, 1996;

Correspondence: Current address: Dr M Kozielska, Department of Pharmacokinetics, Toxicology and Targeting, University of Groningen, Antonius Deusinglaan 1, PO Box 196, Groningen 9700 AD, The Netherlands.

E-mail:m.a.kozielska@rug.nl

Received 8 March 2009; revised 1 July 2009; accepted 13 July 2009; published online 12 August 2009
Van Boven and Weissing, 1998; Jaenike, 1999; Montchamp-Moreau et al., 2001; Atlan et al., 2003).

The presence of a driving chromosome is usually not neutral with respect to individual fitness, both in heterozygous and homozygous condition (for example, see Wallace, 1948; Curtsinger and Feldman, 1980; Jaenike, 1996; Taylor and Ingvarsson, 2003; Atlan et al., 2004). In some well-studied cases, homozygosity for a distorter allele causes sterility in males, or even lethality in males and females (for example, the $t$-complex of the house mouse and the segregation distorter of Drosophila melanogaster; see Lyttle, 1991; Burt and Trivers, 2006). Therefore, there will often be strong selection for suppressors of segregation distortion (for example, see Hurst et al., 1996). Such suppressors have been indeed found in most of the species harbouring segregation distorters (for example, see Jaenike, 2001; Burt and Trivers, 2006).

When segregation distorters are located on sex chromosomes, they not only have an effect on individual fitness but also lead to biased sex ratios in the population (Jaenike, 2001). Biased sex ratios induce selection for increased production of the rarer sex. Unbiased sex ratios can either be restored through suppressors of drive or through a change in the mechanism of sex determination (Bull and Charnov, 1977; Cosmides and Tooby, 1981; Werren and Beukeboom, 1998; Burt and Trivers, 2006). Segregation distortion has been proposed as the driving force behind a change in the sex-determining mechanism of the wood lemming, Myopus schisticolor (Bengtsson, 1977), the mole, Talpa occidentalis (McVean and Hurst, 1996), the creeping vole, Microtus oregoni (Charlesworth 
and Dempsey, 2001), the sciarid fly, Sciara coprophila (Haig, 1993b), the housefly, Musca domestica (Clark, 1999) and scale insects, Neococcoidea (Haig, 1993a).

To our knowledge, there are only very few mathematical models investigating the effect of sex chromosome segregation distortion on the evolution of sex determination. Present models are tailored to a particular species, and aim to explain the observed sex-determining mechanism in that species (Bengtsson, 1977; Jayakar, 1987; McVean and Hurst, 1996; Charlesworth and Dempsey, 2001). Therefore, there is currently still little understanding of the conditions under which sex chromosome segregation distortion can lead to changes in sex-determining mechanisms. We aim to fill this gap by means of a more generic model in which we analyse the conditions for the spread of a new sex-determining gene in a system with a distorting (driving) sex chromosome.

The best documented and probably most common case is segregation distortion occurring during the production of male gametes, wherein it is manifested by the dysfunction of sperm (or pollen) lacking the driving element, or, more precisely, loss of gametes carrying the sensitive allele (for example, see Lyttle, 1993; Taylor and Ingvarsson, 2003). Sex chromosomal distortion is most common in systems with male heterogamety, and usually, the $\mathrm{X}$ chromosome drives against the $\mathrm{Y}$ chromosome. Presumably, Y drive is less common, because it easily leads to population extinction (Hamilton, 1967; Taylor and Ingvarsson, 2003). Therefore, we chose to focus on the case in which the initial sex-determining mechanism is an $X Y$ system and in which segregation distortion occurs only in males.

We consider three scenarios: a driving $X$ chromosome (scenario 1); a driving Y chromosome (scenario 2); and a driving autosome with a male-determining factor (scenario 3). Segregation distortion associated with all such chromosomes has been found in natural populations of various species (Clark, 1999; Jaenike, 2001; Burt and Trivers, 2006). The presence of driving chromosomes leads to female-biased (scenario 1) or male-biased (scenarios 2 and 3) sex ratios, presumably promoting the spread of new masculinizing or feminizing factors, respectively. Throughout, we assume that a distorting allele has a detrimental effect on fitness in homozygous condition, either by reducing male fertility or by reducing viability in males and/or females.

By means of a dynamic model, we address the following questions. Under which circumstances does sex chromosomal segregation distortion lead to changes in the mechanism of sex determination? More specifically, how is the invasion prospect of a new sexdetermining factor affected by the strength of distortion and the fitness effect of a distorter present in the population? When invasion is possible, will a new factor spread to fixation, leading to a switch to a different sexdetermining mechanism? How is the frequency of the segregation distorter affected by the invasion of a new sex-determining factor?

The model

We model the evolutionary dynamics of the sexdetermining system with a set of recurrence equations. We assume an infinite diploid population with random mating and non-overlapping generations. We analyse three scenarios in an initial $X Y$ system: (1) a driving $X$ chromosome, (2) a driving Y chromosome and (3) a driving autosomal male-determining factor. First, we will present our general model assumptions and then introduce modifications specific to the three scenarios.

\section{Genotypes and sex determination}

As the number of ways in which sex could be determined is virtually unlimited, we decided to base our model on a relatively general mode of sex determination. We consider a sex-determination system consisting of three independent gene loci (on three different chromosomes). In the absence of segregation distortion, each locus has two basic alleles, but additional alleles can be present in specific models (see below). The first locus corresponds to the standard XY system of sex determination with two basic alleles: $X$ and $Y$, in which $Y$ corresponds to a dominant male-determining factor. As $\mathrm{X}$ drive leads to female-biased sex ratios, one might expect selection in favour of male-determining factors. Therefore, we also consider a second locus, which harbours a dominant male-determining $M$ allele and a standard $m$ allele. We assume that this locus is autosomal (not linked to the $\mathrm{X}$ or $\mathrm{Y}$ chromosome). Such autosomal male-determining factors have been found in a number of species (Martin et al., 1980; Traut and Willhoeft, 1990; Dübendorfer et al., 2002), and are a likely step in the evolution of sex-determining mechanisms (Bull, 1983). Y chromosomal drive leads to male-biased sex ratios and should favour feminizing genes. Therefore, we also consider a third locus, which has a female-determining $F$ allele and a standard $f$ allele. There are many possibilities of how female and male-determining factors interact during sexual development. We consider the simplest case, in which $F$ is dominant over $M$ and $Y$, meaning that the presence of $F$ always leads to female development, even if both $Y$ and $M$ are present in homozygous state. We assume that the lack of an $X$ or $Y$ chromosome does not have negative effects on fitness (see Discussion). If $F$ is absent, but $Y$ and/or $M$ are present, an individual becomes a male, otherwise (neither $Y$ nor $M$ is present) it becomes a female. All possible male and female genotypes are summarized in Table 1. This sex-determining system resembles that of the housefly (Dübendorfer et al., 2002; Kozielska et al., 2006), but we believe that the model is more generally applicable.

Table 1 All possible genotypes considered in the model (without distinguishing between driving and non-driving alleles)

\begin{tabular}{llllll}
\hline \multicolumn{3}{c}{ Females } & & & Males \\
\hline$X X$ & $m m$ & $f f$ & $X Y$ & $m m$ & $f f$ \\
$X X$ & $m m$ & $F f$ & $X Y$ & $M m$ & $f f$ \\
$X X$ & $M m$ & $F f$ & $X Y$ & $M M$ & $f f$ \\
$X X$ & $M M$ & $F f$ & $X X$ & $M m$ & $f f$ \\
$X Y$ & $m m$ & $F f$ & $X X$ & $M M$ & $f f$ \\
$X Y$ & $M m$ & $F f$ & $Y Y$ & $m m$ & $f f$ \\
$X Y$ & $M M$ & $F f$ & $Y Y$ & $M m$ & $f f f$ \\
$Y Y$ & $m m$ & $F f$ & $Y Y$ & $M M$ & $f f$ \\
$Y Y$ & $M m$ & $F f$ & & & \\
$Y Y$ & $M M$ & $F f$ & & & \\
\hline
\end{tabular}


Drive

Segregation is random in females and in males that do not possess a driving chromosome or are homozygous for it. In males heterozygous for a driving chromosome and sensitive chromosomes (also see below), $k$ denotes the frequency of the driving allele in gametes and, hence, indicates the strength of distortion.

\section{Fitness}

As described in the Introduction, the presence of distorter allele is often detrimental for fitness. Our invasion analysis (Appendix B) is based on quite general assumptions on the fitness effects of a driving allele in homozygous condition: homozygous males have fertility $u(0 \leqslant u \leqslant 1)$, and the viability of homozygous males and females is given by $v_{m}$ and $v_{f}$, respectively $\left(0 \leqslant v_{m}, v_{f} \leqslant 1\right)$. For the numerical analysis, which allows us to study the full dynamics of the system, we considered three, more specific, fitness schemes: (a) no cost of drive; (b) males homozygous for the driving allele (that is $X^{\mathrm{d}} X^{\mathrm{d}}, Y^{\mathrm{d}} Y^{\mathrm{d}}$ or $M^{\mathrm{d}} M^{\mathrm{d}}$, depending on the scenario) are sterile; and (c) homozygosity for the driving allele is lethal, both in males and females.

\section{Evolutionary dynamics}

We assume random mating and discrete non-overlapping generations. Under these conditions, the dynamics of the system can be easily expressed by a set of recursion equations for the frequency of each genotype in each generation, depending on the frequency of all genotypes in the previous generation (see Appendix A). Iteration of these equations allows studying the dynamics of the system.

In our numerical iterations, we started with the standard $X Y$ system (females: $X X ; m m$; ff and males: $X Y ; m m ; f f)$, and at generation 0 , introduced a distorter allele (step 1) at a frequency of 0.001 . We let the system evolve and when the equilibrium was reached, a new sex-determining factor was introduced (step 2) at a frequency of 0.001. Again, frequencies of different genotypes were calculated at every generation until a new equilibrium appeared to be reached. All simulation results were confirmed by a mathematical invasion analysis (see Appendix B for details).

The three scenarios for sex chromosomal distortion were implemented as follows:

\section{Scenario 1: driving $X$ chromosome}

In this version of the model, three alleles segregate at the XY locus: standard $Y$, standard $X$ and driving $X$. The latter will be denoted $X^{\mathrm{d}}$, and it is assumed to drive only against $Y$. Therefore, the frequency of $X^{\mathrm{d}}$ gametes produced by $X^{\mathrm{d}} Y$ males is equal to the drive strength $k$ and the frequency of $Y$ gametes equal $1-k$. For all other male genotypes, segregation of alleles is Mendelian $(k=1 / 2)$. We start with the standard XY system, and in step 1 (see above), introduce a driving $X^{\mathrm{d}}$ chromosome. This causes female-biased sex ratios, and in step 2, an $M$ allele is introduced to regain equal sex ratios.

\section{Scenario 2: driving $Y$ chromosome}

In this version, three alleles segregate at the $X Y$ locus: standard $X$, standard $Y$ and driving $Y$. The later will be denoted $Y^{\mathrm{d}}$ and it is assumed to drive only against $X$. Therefore, $X Y^{\mathrm{d}}$ males produce $X$ gametes in frequency $1-k$ and $Y^{\mathrm{d}}$ gametes in frequency $k$. For all other male genotypes, segregation of alleles is Mendelian $(k=1 / 2)$. We start with the standard $X Y$ system, and in step 1 , introduce a driving $Y^{\mathrm{d}}$ chromosome. This causes male-biased sex ratios, and in step 2 , an $F$ allele is introduced.

\section{Scenario 3: driving $M$}

In this scenario, segregation occurs at an autosomal locus, in which $M$ drives against $m$. The driving alleles will be denoted as $M^{\mathrm{d}}$. The frequency of $M^{\mathrm{d}}$ in the gametes of $M^{\mathrm{d}} m$ males is equal to $k$, and the frequency of $m$ gametes equals $1-k$. For all other male genotypes, segregation of alleles is Mendelian $(k=1 / 2)$. We start with the standard XY system, and in step 1, introduce a driving $M^{\mathrm{d}}$ allele. This causes male-biased sex ratios, and in step 2, an $F$ allele is introduced.

For each scenario, we run numerical iterations of the recursion equations (Appendix $A$ ) to investigate the effects of distortion strength $k$ and various fitness schemes on the dynamics of the system. The results of the numerical analysis are fully in line with the (much more general) mathematical invasion analysis given in the Appendix B.

\section{Results}

\section{Scenario 1: driving $X$ chromosome}

For any value of $k>\frac{1}{2}$, the driving $X$ chromosome $\left(X^{\mathrm{d}}\right)$ invades the population, leading to a female-biased sex ratio. When $M$ is introduced, it increases the production of males, and it is selected for, as long as selection against biased sex ratios is not overcome by selection against sterile genotypes. Once $M$ invades, it causes the loss of $Y$ from the population and restores the equal sex ratio. Therefore, in most cases, there is a switch from an XY system to an XX system, in which sex is determined by $M$. Males are again the heterogametic sex, therefore, a new system can be seen as male heterogamety for $M$ (Table 2). Interestingly, even though the presence of the driving $X^{\mathrm{d}}$ chromosome is often responsible for the switch from $X Y$ to an autosomal system, at equilibrium, the $X^{\mathrm{d}}$ chromosome may be no longer present in the population (see below).

No cost of drive: Owing to its transmission advantage and no fitness costs, $X^{\mathrm{d}}$ fixates in the population, removing the standard $X$ chromosome independently of the value of $k$. This leads to a female-biased sex ratio with the proportion of females equal to the drive strength $k$. This sex ratio bias facilitates the invasion of $M$, which very quickly spreads replacing $Y$ as a sexdetermining factor, leading to male heterogamety for $M$ (Figure 1a). $X^{\mathrm{d}}$ reaches fixation in both males and females. Although driving chromosomes are still present in the population, the sex ratio is unbiased, as the chromosomes sensitive to drive are absent.

Sterility of $X^{\mathrm{d}} X^{\mathrm{d}}$ males: The $X^{\mathrm{d}}$ invades the population and replaces the standard $X$, as above, as males are always $X^{\mathrm{d}} Y$ and, therefore, do not have a fertility disadvantage. However, now the introduction of $M$ 
Table 2 Overview of changes in the sex-determining system induced by a driving $X$ chromosome $\left(X^{\mathrm{d}}\right)$

\begin{tabular}{|c|c|c|c|c|}
\hline & No cost of drive & \multicolumn{2}{|c|}{ Sterility of $\mathrm{X}^{d} \mathrm{X}^{d}$ males } & Lethality of $\mathrm{X}^{d} \mathrm{X}^{d}$ individual \\
\hline Initial state & & \multicolumn{2}{|c|}{$\begin{array}{c}\text { o } X Y ; m m ; f f \\
\text { o XX; } m m ; f f \\
\hat{S}=\frac{1}{2}\end{array}$} & \\
\hline After introduction of $X^{d}$ & & $\begin{array}{c}\hat{o} X^{\mathrm{d}} Y ; m m ; f f \\
\text { o } X^{\mathrm{d}} X^{\mathrm{d}} ; m m ; f f \\
\hat{S}=1-k\end{array}$ & & $\begin{array}{c}\text { ô } X^{\bullet} Y ; m m ; f f^{\mathrm{a}} \\
\text { † } X^{\bullet} X ; m m ; f f^{\mathrm{a}} \\
\quad \frac{2}{5}<\hat{S}<\frac{1}{2}\end{array}$ \\
\hline After introduction of $M$ & $\begin{array}{c}\hat{o} X^{\mathrm{d}} X^{\mathrm{d}} ; M m ; f f \\
\text { o } X^{\mathrm{d}} X^{\mathrm{d}} ; m m ; f f \\
\hat{S}=\frac{1}{2}\end{array}$ & $\begin{array}{c}k \leqslant \frac{3}{4} \\
\text { o } X^{\mathrm{d} Y} ; m m ; f f \\
\text { ᄋ } X^{\mathrm{d}} X^{\mathrm{d}} ; m m ; f f \\
\hat{S}=1-k\end{array}$ & $\begin{array}{c}k>\frac{3}{4} \\
\text { ô } X X ; M m ; f f \\
\text { ᄋ XX; } \quad x m ; f f \\
\hat{S}=\frac{1}{2}\end{array}$ & $\begin{array}{c}\text { ๙ XX;Mm;ff } \\
\text { o XX;mm;ff } \\
\hat{S}=\frac{1}{2}\end{array}$ \\
\hline
\end{tabular}

Resulting system

Male heterogamety for $M \quad$ Male heterogamety for $Y$ Male heterogamety for $M$

Male heterogamety for $M$

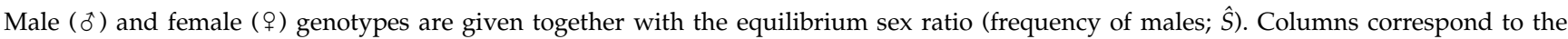
different fitness schemes considered (top row). When the outcome is the same for two fitness schemes, the corresponding columns are merged. Sometimes the outcome depends on the drive strength $(k)$. In that case, different outcomes are listed separately; otherwise the outcome is independent of $k$. The rows correspond to the sequence of events, from the initial state to the equilibrium state after the introduction of driving $X^{\mathrm{d}}$ and the final equilibrium after the introduction of $M$. After the introduction of $M$, in some cases, the standard $X$ chromosome has an advantage over $X^{\mathrm{d}}$ and it will invade the population when reintroduced. Therefore, at equilibrium it will be present in the population. See text for details.

${ }^{\mathrm{a}} \mathrm{X}^{\bullet}$ stands for either standard $X$ or driving $X^{\mathrm{d}}$, as polymorphism is maintained in the population.

leads to the production of sterile $X^{\mathrm{d}} X^{\mathrm{d}}$ males and $M$ cannot invade the population. Only if the standard $X$ is reintroduced into the population together with $M$ can they both invade, as some males produced by $M$ possess the standard $X$ and are fertile. These males have an advantage in strongly female-biased populations, and both $M$ and standard $X$ increase in frequency. An increase in the frequency of $M$ leads to the production of sterile $X^{\mathrm{d}} X^{\mathrm{d}}$ males and selection against $X^{\mathrm{d}} \cdot X^{\mathrm{d}}$ is eventually lost from the population and replaced by standard $X$ (Figure 1b). $Y$ is also removed from the population, and a male heterogametic system for $M$ establishes. It should be noted that, although the driving $\mathrm{X}$ chromosome induced changes in the sexdetermining mechanism, it is no longer present in the population.

This happens only for strong drive $k>\frac{3}{4}$, as this will lead to the population sex ratio being very female biased and selection against the biased sex ratio is strong enough to overcome selection against infertile males. Therefore, even though the presence of $M$ initially leads to the production of many sterile $X^{\mathrm{d}} X^{\mathrm{d}}$ males, if $X$ is present in the population, this cost is compensated for by the increased production of males thanks to $M$. For weaker drive, selection against biased sex ratio is not strong enough to overcome selection against sterile genotypes and $M$ cannot invade (Table 2).

Lethality of $X^{\mathrm{a}} X^{\mathrm{d}}$ genotypes: The $X^{\mathrm{d}}$ invades the population, but it cannot fixate, as homozygous genotypes are lethal. Therefore, the sex ratio bias induced by $X^{\mathrm{d}}$ is less pronounced than that for the scenarios presented above (Figure 1c). The frequency of $X^{\mathrm{d}}$ is equal to $\frac{1}{2}-\frac{1}{4 k}$ (that is, equals $\frac{1}{2}$ for $k=\frac{1}{2}$, and $\frac{1}{4}$ for $k=1$ ). A female-biased sex ratio facilitates the invasion of the masculinizing factor $M$, which in turn leads to a decrease in the frequency of $Y$ and increased homozygosity for $X^{\mathrm{d}}$; also in males. As the standard $X$ is present in the population at high frequency, the decrease in fitness of $M$-bearing males through the production of rare lethal $X^{\mathrm{d}} X^{\mathrm{d}}$ offspring is outweighed by the advantage from an increased production of males. Eventually $X^{\mathrm{d}}$ is removed from the population because of its detrimental effect in the homozygous state, which is not counterbalanced by a transmission advantage, as $X^{\mathrm{d}} Y$ males are rare. A male heterogametic system for $M$ gets established (Table 2).

\section{Scenario 2: driving $\mathrm{Y}$ chromosome}

Independent of the strength of drive $k$ and the fitness of homozygous $Y^{\mathrm{d}} Y^{\mathrm{d}}$ individuals, $Y^{\mathrm{d}}$ always spreads in the population replacing standard $Y$, as females never harbour $Y^{\mathrm{d}}$ and, therefore, males can never be homozygous for $Y^{\mathrm{d}}$. As all males are $X Y^{\mathrm{d}}$, the sex ratio is biased with a proportion of males equal to the drive strength, $k$. Whether $F$ invades depends on the fitness costs of homozygosity for $Y^{\mathrm{d}}$ (Table 3 ). However, similar to scenario 1 , a change in the sex-determining mechanism may lead to the complete removal of the driving chromosome from the population.

No cost of drive: The feminizing $F$ allele always spreads in the population, leading to fixation of $Y^{\mathrm{d}}$ and a switch to a female heterogametic sex-determining system. Although $Y^{\mathrm{d}}$ is present in the population, the sex ratio equals 1:1, as the $X$ chromosome sensitive to drive is lost.

Sterility of $Y^{\mathrm{d}} Y^{\mathrm{d}}$ males: Selection against biased sex ratios facilitates the spread of $F$. It is present only in females, hence it does not have direct negative fitness effects. However, $F$ females produce some sterile $Y^{\mathrm{d}} Y^{\mathrm{d}}$ males, which prevent $F$ from fixation. Polymorphism for $X$ and $Y^{\mathrm{d}}$, and $F$ and $f$ is maintained, but the sex ratio in the population equals 0.5 (Figure 2). The frequencies of $F$ and $Y^{d}$ increase with the strength of drive, $k$. At this state, reintroduction of the standard $Y$ in the population will lead to its spread, as it does not cause a fitness cost in 
104
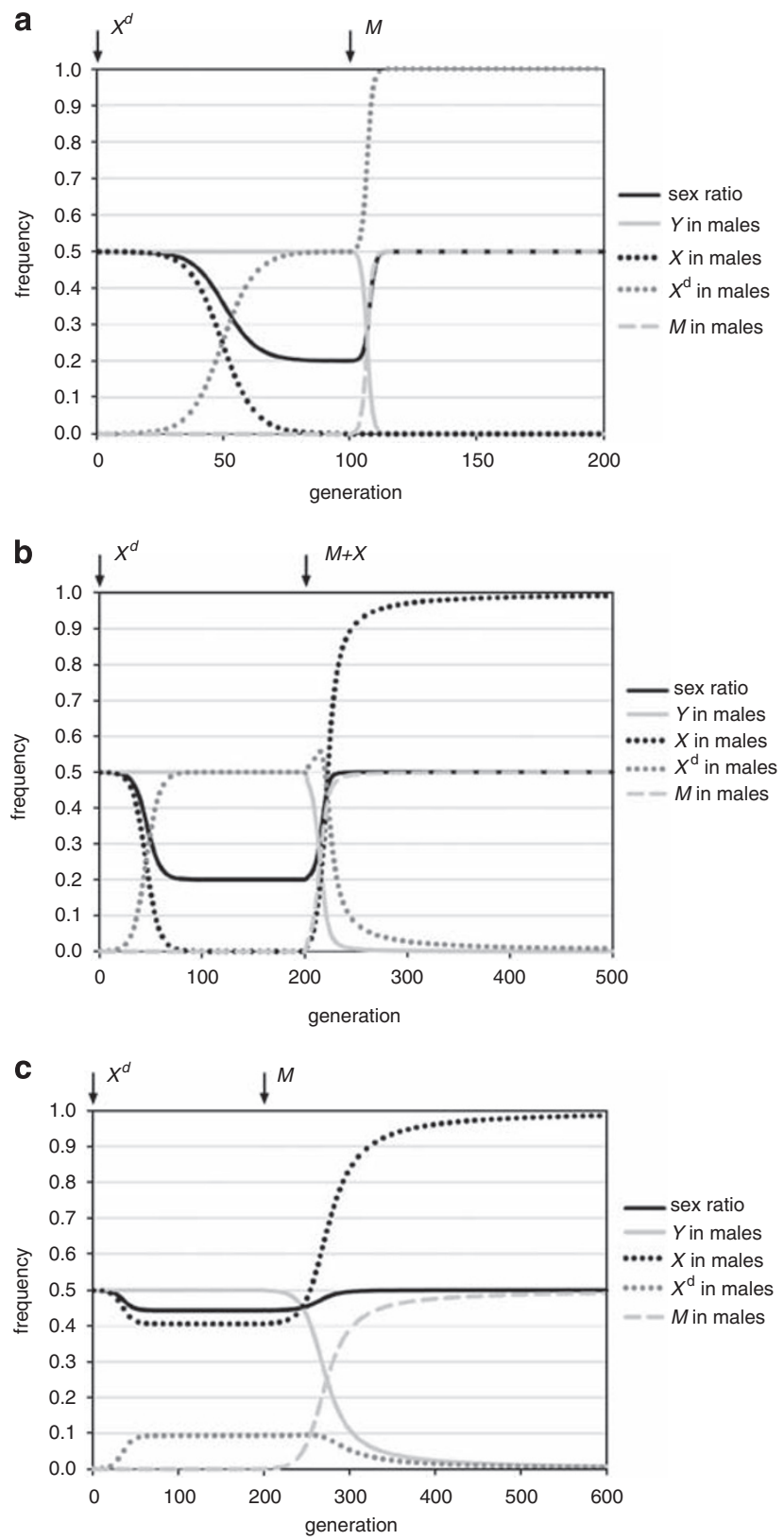

Figure 1 Dynamics of the sex-determining system in the presence of a driving $X^{\mathrm{d}}$ chromosome. This is a representative example with strength of drive $k=0.8$. Introduction of new alleles is indicated by arrows. The spread of $X^{\mathrm{d}}$ leads to biased sex ratios. When the system appeared to reach equilibrium, an autosomal masculinizing factor $M$ was introduced, leading to a switch in sex-determining mechanism to male heterogamety for $M$ and restoration of equal sex ratios. (a) No cost of drive. Standard $X$ and $Y$ are lost from the population. (b) Sterility of $X^{\mathrm{d}} X^{\mathrm{d}}$ males. A driving $X^{\mathrm{d}}$ leads to the loss of standard $X$. In generation, $200 M$ and $X$ are introduced simultaneously, $X^{\mathrm{d}}$ and $Y$ disappear from the population. Introducing $M$ on its own has no effect (see Results). (c) Lethality of $X^{d} X^{d}$ genotypes. Initially polymorphism for $X^{\mathrm{d}}$ and $X$ is maintained. Introduction of $M$ leads to the removal of $X^{\mathrm{d}}$ and $Y$.

males. The segregation advantage of $Y^{\mathrm{d}}$ is low, as owing to the low frequency of $X$, the frequency of $Y^{d} X$ males is low. Therefore, the segregation advantage is outweighed by selection against sterile $Y^{\mathrm{d}} Y^{\mathrm{d}}$ males. The frequency of $Y^{\mathrm{d}}$ decreases, and standard $Y$ replaces $Y^{\mathrm{d}}$ and spreads to fixation. $X$ is removed, $F$ is present in all females and a female heterogametic system establishes (Figure 2).

Lethality of $Y^{d} Y^{d}$ genotypes: If drive strength is low, $F$ does not invade the population, as selection against a (slightly) biased sex ratio is weaker than selection against the lethal genotypes produced when $F$ is present. Therefore, an $X Y$ system with the driving $Y^{\mathrm{d}}$ chromosome (that is, no standard $Y$ ) and a biased sex ratio is stable for $\mathrm{k}<\frac{3}{5}$ (see Appendix B). For higher values of $k$, the $F$ factor invades the population. Initially, a polymorphism on both the $F$ and $X Y$ loci is maintained, with a population sex ratio that is only slightly male biased, similar to the scheme with male sterility. For $k$ between $\frac{3}{5}$ and 0.655 (see Appendix B), this polymorphic sex-determining system is stable and $Y$ cannot reinvade the population. However, for $k>0.665$, the reintroduction of the standard $Y$ also leads to an increase of $F$ in frequency and eventually to removal of $Y^{\mathrm{d}}$ and $X$. $F$ reaches a frequency of $\frac{1}{2}$ in females and female heterogamety with equal sex ratios is established.

When $F$ is present in the population, but before fixation of standard $Y$, in some circumstances, autosomal $M$ can invade. However, it never reaches fixation, and standard $Y$ will fixate in the population once it is reintroduced. Therefore, female heterogamety with the frequency of standard $Y$ equal to 1 is an equilibrium state of this system, although polymorphism for $M$ and $m$ can be present.

\section{Scenario 3. driving autosomal $M$}

Similar to the case with driving $Y^{\mathrm{d}}$, driving $M^{\mathrm{d}}$ invades the population regardless of drive strength and the fitness of individuals homozygous for $M^{\mathrm{d}}$, as it is only present in males in heterozygous state. $Y$ is removed from the population and all individuals are $X X$. The population sex ratio is male biased and equal to drive strength, $k$. Whether $F$ invades depends on the fitness costs of homozygosity for $M^{\mathrm{d}}$ (Table 4 ).

No cost of drive: For any drive strength $k$, selection against male-biased sex ratios facilitates the spread of the feminizing $F$ allele. The invasion of $F$ leads to fixation of $M^{\mathrm{d}}$, and a switch to a female heterogametic system with 1:1 sex ratio.

Sterility of $M^{\mathrm{d}} M^{\mathrm{d}}$ males: $F$ always invades irrespective of drive strength. However, fixation of $F$ and $M^{\mathrm{d}}$ is impossible, as this will lead to all males being sterile. Therefore, there is no full switch to female heterogamety, but polymorphism for both $M^{\mathrm{d}}$ and $m$, and $F$ and $f$ is maintained in the population. The population sex ratio equals 0.5 . With increasing $k$, the equilibrium frequency of $M^{\mathrm{d}}$ and $F$ increases. If at any point $Y$ is reintroduced into this system, it will reinvade the population, as it assures maleness without fitness costs. Eventually, $Y$ spreads to fixation and all females become heterozygous for $F$. The frequency of $M^{\mathrm{d}}$ decreases, but it is not removed from the population. Therefore, at the stable equilibrium, in effect, a female heterogametic system is present, but with a polymorphism at the $M$ locus (Figure 3).

Lethality of $M^{\mathrm{d}} M^{\mathrm{d}}$ genotypes: If the drive strength is weak $\left(k<\frac{3}{5}\right)$, then the population sex ratio is only slightly 
Table 3 Overview of changes in the sex-determining system induced by a driving $Y$ chromosome $\left(Y^{\mathrm{d}}\right)$

\begin{tabular}{|c|c|c|c|c|c|}
\hline & No cost of drive & Sterility of $\mathrm{Y}^{d} \mathrm{Y}^{d}$ males & \multicolumn{3}{|c|}{ Lethality of $\mathrm{Y}^{d} \mathrm{Y}^{d}$ individuals } \\
\hline Initial state & & & $\begin{array}{l}\hat{o} X Y ; m m ; f f \\
\text { ᄋ XX;mm;ff } \\
\hat{S}=\frac{1}{2}\end{array}$ & & \\
\hline After introduction of $Y^{\mathrm{d}}$ & & & $\begin{array}{c}\text { o } X Y^{\mathrm{d}} ; m m ; f f \\
\text { o XX;mm;ff } \\
\hat{S}=k\end{array}$ & & \\
\hline After introduction of $F$ & $\begin{array}{c}\hat{o} Y^{\mathrm{d}} Y^{\mathrm{d}} ; m m ; f f \\
\text { o } Y^{\mathrm{d}} Y^{\mathrm{d}} ; m m ; F f \\
\quad \hat{S}=\frac{1}{2}\end{array}$ & $\begin{array}{c}\text { o } Y Y ; m m ; f f \\
\text { o } Y Y ; m m ; F f \\
\hat{S}=\frac{1}{2}\end{array}$ & $\begin{array}{c}k \leqslant \frac{3}{5} \\
\text { ๙ } X Y^{\mathrm{d}} ; m m ; f f \\
\text { OXX;mm;ff } \\
\hat{S}=k\end{array}$ & $\begin{array}{c}\frac{3}{5}<k \leqslant 0.655 \\
\delta^{x^{*} Z^{\bullet} Y^{d} ; m m ; f f} \\
\text { ᄋ } Z^{\bullet} Z^{\bullet} ; m m ; F^{\bullet} f \\
\frac{1}{2}<\hat{S}<k\end{array}$ & $\begin{array}{c}k>0.665 \\
\text { o } Y Y ; m m ; f f \\
\text { क } Y Y ; m m ; F f \\
\quad \hat{S}=\frac{1}{2}\end{array}$ \\
\hline Resulting system & Female heterogamety & Female heterogamety & Male heterogamety & Polymorphism & Female heterogamety \\
\hline
\end{tabular}

Data are organized as in Table $2 . F^{\bullet}$ represents either $F$ or $f$. Hence $F^{\bullet} f$ indicates presence of both $F f$ or $f f$ genotypes in females. $Z^{\bullet}$ represents either $X$ or $Y^{\mathrm{d}}$. After the introduction of $F$, in some cases, the standard, non-driving $Y$ chromosome has an advantage over $Y^{\mathrm{d}}$ and it will invade the population when reintroduced. See text for details.

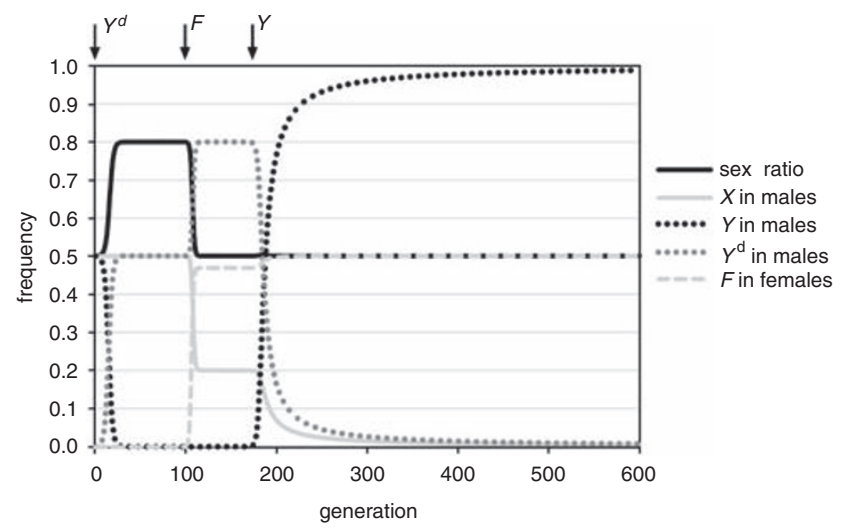

Figure 2 Dynamics of the sex-determining system with a driving $Y^{\mathrm{d}}$ chromosome for the case in which $Y^{\mathrm{d}} Y^{\mathrm{d}}$ males are sterile. Introduction of new alleles is indicated by arrows. $Y^{\mathrm{d}}$ (with drive strength $k=0.8$ ) replaces standard $Y$ and leads to a strongly malebiased sex ratio. Introduction of $F$ leads to a polymorphic system. If standard $Y$ is reintroduced into this system, it invades the population and fixates, replacing the driving $Y^{\mathrm{d}}$ and leading to a female heterogametic system.

male-biased and $F$ does not invade, because the advantage of producing females by $F$ is outweighed by the disadvantage of producing lethal, homozygous $M^{\mathrm{d}}$ individuals. Therefore, for weak drive, a system with male heterogamety for $M^{\mathrm{d}}$ and a biased sex ratio is stable. For higher $k, F$ invades, leading to a system polymorphic for $F$ and $f$, and $M^{\mathrm{d}}$ and $m$, with the equilibrium frequency of $F$ and $M^{\mathrm{d}}$ increasing with $k$ (as it does in the scheme with sterile males). The population sex ratio is slightly male biased. For $k$ between $\frac{3}{5}$ and 0.8 (see Appendix B), this polymorphic sex-determining system is stable and $Y$ cannot reinvade the population. For stronger drive $(k \geqslant 0.8)$, if $Y$ is reintroduced it will spread to fixation, leading to female heterogamety. Although the frequency of $M^{\mathrm{d}}$ decreases, it is not removed from the population and polymorphism at the $M$ locus is maintained (similar to the case with sterility of $M^{\mathrm{d}} M^{\mathrm{d}}$ males).

\section{Discussion}

Sex determination is a fundamental developmental process and one might expect sex-determining mechanisms to be highly stable. Yet, sex-determining mechanisms vary greatly between different taxonomic groups and even between closely related species (Bull, 1983; Kraak and Pen, 2002; Janzen and Phillips, 2006). Although steady empirical progress is being made in unravelling the genetics of sex-determining mechanisms, there are still many unanswered questions about how new sex-determining systems evolve. It has been proposed that 'genetic conflict is the most likely general explanation for the diversity of sex-determining mechanisms' (Werren and Beukeboom, 1998). Recent theoretical work has largely focused on maternal-offspring conflict over sex ratio (reviewed in Uller et al., 2007).

Intragenomic conflict (between different genes within an individual), in general, and segregation distortion, in particular, is another selective force that can lead to changes in sex-determining systems (see Introduction). However, there is little theoretical understanding of the process. Most models considered thus far were tailored to one particular species and aimed at explaining the sex-determining mechanisms found in that species (Bengtsson, 1977; McVean and Hurst, 1996). The more general models make some unrealistic assumptions. For example, Jayakar (1987) assumed that the driving chromosome shows the same segregation distortion in males and females. Charlesworth and Dempsey (2001) assumed that the distorter has a complete transmission advantage (present in 100\% of gametes) in females and a complete transmission disadvantage in males.

In this paper, we developed some general models to investigate the change in the mechanisms of sex determination in systems in which a driving sex chromosome is present. Although trying to be as general as possible, we had to make some assumptions concerning sex determination and segregation distortion. Although we believe that our assumptions reflect the most common patterns observed in natural systems (see the Introduction), we briefly discuss how our results might be affected if these assumptions were not met. 
Table 4 Overview of changes in the sex determining system induced by a driving autosomal factor $M^{\mathrm{d}}$

\begin{tabular}{|c|c|c|c|c|c|}
\hline & No cost of drive & Sterility of $\mathrm{M}^{d} \mathrm{M}^{d}$ males & \multicolumn{3}{|c|}{ Lethality of $\mathrm{M}^{d} \mathrm{M}^{d}$ individuals } \\
\hline Initial state & & & $\begin{array}{c}\hat{o} X Y ; m m ; f f \\
\text { } \mathrm{XXX} ; m m ; f f \\
\hat{S}=\frac{1}{2}\end{array}$ & & \\
\hline After introduction of $M^{\mathrm{d}}$ & & & $\begin{array}{c}\text { o XX; } M^{\mathrm{d}} m ; f f \\
\text { o XX;mm;ff } \\
\hat{S}=k\end{array}$ & & \\
\hline After introduction of $F$ & $\begin{array}{c}\text { ○ } X X ; M^{\mathrm{d}} M^{\mathrm{d}} ; f f \\
\text { ᄋ } X X ; M^{\mathrm{d}} M^{\mathrm{d}} ; F f \\
\hat{S}=\frac{1}{2}\end{array}$ & $\begin{array}{c}\text { ô } Y Y ; M^{\bullet} M^{\bullet} ; f f \\
\text { क } Y Y ; M^{\bullet} M^{\bullet} ; F f \\
\quad \hat{S}=\frac{1}{2}\end{array}$ & $\begin{array}{c}k \leqslant \frac{3}{5} \\
\text { o } X X ; M^{\mathrm{d}} m ; f f \\
\text { o XX;mm;ff } \\
\quad \hat{S}=k\end{array}$ & $\begin{array}{l}\quad \frac{3}{5}<k<0.8 \\
\text { o } X X ; M^{\bullet} m ; f f \\
\text { क XX; } M^{\bullet} m ; F^{\circ} f \\
\\
\quad \frac{1}{2}<\hat{S}<k\end{array}$ & $\begin{array}{c}\quad k \geqslant 0.8 \\
\text { ô } Y Y ; M^{\bullet} m ; f f \\
\text { क } Y Y ; M^{\bullet} m ; F f \\
\quad \hat{S}=\frac{1}{2}\end{array}$ \\
\hline
\end{tabular}

Resulting s.d. system Female heterogamety Female heterogamety Male heterogamety Polymorphism Female heterogamety

Data organized as in Table 2. $M^{\bullet}$ represents either $m$ or driving $M^{\mathrm{d}}$. Hence $M^{\bullet} M^{\bullet}$ indicates a polymorphism on this locus and the presence of $m m, m M^{\mathrm{d}}$ and $M^{\mathrm{d}} M^{\mathrm{d}}$ genotypes. $F^{\bullet}$ represents either $F$ or $f$. Hence $F^{\bullet} f$ indicates presence of both $F f$ or $f f$ genotypes in the population.

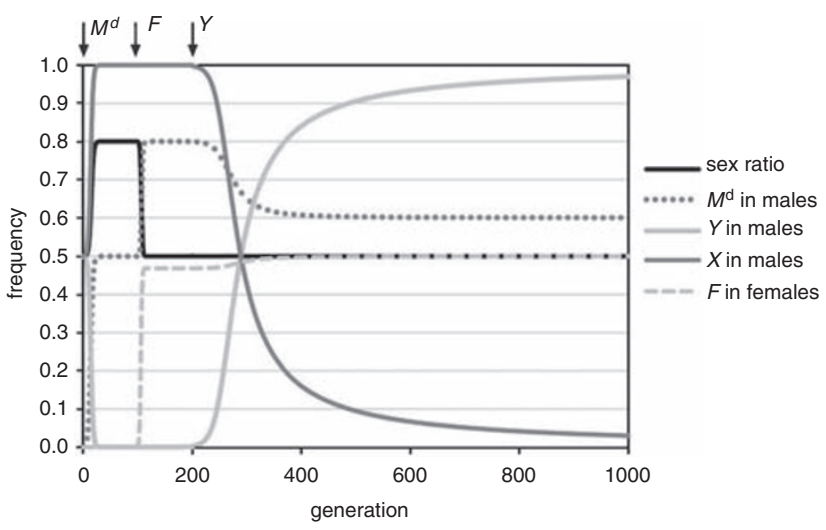

Figure 3 Dynamics of the sex-determining system with a driving autosomal $M^{\mathrm{d}}$ factor for the case of sterility in $M^{\mathrm{d}} M^{\mathrm{d}}$ males. Introduction of new alleles is indicated by arrows. Spread of $M^{\mathrm{d}}$ (with drive strength $k=0.8$ ) leads to the removal of $Y$, the switch to a male heterogametic system for $M^{\mathrm{d}}$, and male-biased sex ratios. Introduction of $F$ leads to a polymorphic system. When $Y$ is reintroduced, it leads to a switch to a female heterogametic system, but polymorphism on the $M$ locus is maintained.

In our model, we consider segregation distortion only in males, the heterogametic sex. Male heterogamety is the prevailing sex-determining mechanism in species with genetic sex determination, and most of the described cases of segregation distortion affect sperm or pollen production (Jaenike, 2001; Taylor and Ingvarsson, 2003). However, meiotic drive has also been found during female meiosis (for example, in maize; Buckler et al., 1999), and sex ratio segregation distortion in $X Y$ (ZW) females has been described in wood lemmings (Bengtsson, 1977) and postulated in the butterfly Danaus chrysippus (Smith et al., 1998). For reasons of symmetry, the results of our model immediately carry over to systems with female heterogamety and meiotic drive occurring in females. For example, if females are ZW, and $Z$ drives against $W$ (symmetric to a driving $X$ ) and homozygosity for driving $Z$ causes lethality, a driving $Z$ should invade but not reach fixation, and introduction of an autosomal feminizing gene would lead to a switch to female heterogamety of an autosomal factor and removal of the driving $\mathrm{Z}$ from the population. This is assuming that the autosomal $M$ in our model becomes a feminizing factor and $F$ becomes a masculinizing factor. Our scenario of sterility of males that are homozygous for the driving allele would, by symmetry, apply to cases in which females that are homozygous for the driving allele are sterile. We do not know of such cases in nature, but this scenario seems plausible, taking into account that a driving chromosome disturbs meiosis.

We have performed a rather general invasion analysis of new sex-determining genes for a broad range of fitness schemes. The full dynamics of the system could only be studied numerically, and hence could only be performed for a few specific cases. To this end, we focused on lethality and/or male sterility in individuals homozygous for the driving allele. However, our analytical results (Appendix B) suggest that the numerical analysis can be extrapolated to other fitness schemes as well, as the invasion prospects of a new sex-determining factor do not often depend on the precise fitness values. Examples of reduced fitness of individuals possessing driving alleles, not only in homozygous but also in heterozygous state, or even increased fitness of heterozygous females, are known from nature (for example, Wallace, 1948; Curtsinger and Feldman, 1980; Jaenike, 1996; Atlan et al., 2004; Wilkinson et al., 2006). In general, fitness values of different genotypes will influence the balance between sex ratio selection (favouring spread of a new sex-determining factor) and viability selection acting on new sex-determining factors. Strongly reduced viability may prevent the spread of a new factor. However, according to our results, once new factors invade, the equilibrium sex-determining mechanism is largely independent of the specific fitness effects. This may hold at least as long as segregation distorters have a negative effect on fitness. When distorter alleles show overdominance in females (Wallace, 1948; Curtsinger and Feldman, 1980; Wilkinson et al., 2006), the outcome of evolution may be different, as both fixation and removal of the distorter allele may not be favoured.

In addition, one could imagine that a new sexdetermining gene has fitness costs on its own (for example, because of some genetic incompatibilities), 
which would hamper its spread (Werren and Beukeboom, 1998). Here again, results would probably depend on the balance between selection favouring spread of a new factor (sex ratio bias caused by drive) and selection against it (reduced fitness). A few simulations that we did show was that if a new sex-determining factor has only a slightly reduced fitness (a reduction by 5\%), the resulting system is the same as that with no fitness costs, at least for the case with a driving $\mathrm{Y}$ chromosome. However, a complete switch may not occur if the $M$ factor causes a fitness reduction. Interestingly, in case of lower fitness of $F$, we sometimes observed cycling behaviour. An increase in the frequency of $F$ leads to a decrease in the frequency of $Y^{\mathrm{d}}$, which reduces sex ratio bias and selection favouring $F$, which in turn decreases $F$ frequency, which allows an increase in the frequency of $Y^{d}$, which re-creates a biased sex ratio and increase in the frequency of $F$ and the cycle repeats (results now shown).

In our model, we also assumed that the absence of a $Y$ or $\mathrm{X}$ chromosome does not have negative effects on fitness. This is probably not realistic in species with old, strongly differentiated sex chromosomes, in which $\mathrm{Y}$ usually lacks some of the genes that are present on the $X$ chromosome, or when $\mathrm{Y}$ possesses genes crucial for gametogenesis. In such cases, some of our results will not hold, as a full switch to another sex-determining system requires removal of one of the original sex chromosomes (either $\mathrm{X}$ or $\mathrm{Y}$ ). However, absence of one of the sex chromosomes does not necessarily lead to (drastic) reduction in fitness (Juchault and Rigaud, 1995). Undifferentiated sex chromosomes have been found in a number of animal and plant species (Charlesworth et al., 2005; Fraser and Heitman, 2005), and even if the chromosomes are morphologically differentiated, they may not possess any genes necessary for viability or fertility (Hiroyoshi, 1977). More theoretical and empirical work is needed to understand the effect of different fitness values associated with different genotypes.

The switch to a new sex-determining system is driven by selection against biased sex ratios. When there are no fitness costs associated with homozygosity for the distorter allele, even weak sex ratio selection leads to changes in sex-determining mechanism. However, often sex ratio selection has to be sufficiently strong to overcome selection against less-fit genotypes (Appendix B). Therefore, in some cases, only strong segregation distorters will lead to changes in sex-determining mechanisms. This suggests that when segregation distortion is weak, the evolution of suppressors may be the only way to decrease sex ratio bias. Interestingly, Lyttle (1981) in his model on the evolution of new sex-determining mechanism through aneuploidy, also concluded that strong drive favours change in sex-determining mechanisms and weak drive favours the accumulation of suppressors. We suspect that this is a general phenomenon. Moreover, once suppressors start accumulating in the population decreasing the effective drive, this may prevent changes in the sex-determining system, as long as suppressors are less costly than a new sex-determining mechanism.

According to our model, the invasion prospects of a new sex-determining factor may depend on whether the distorter has any effects on the fertility of males or on the viability of both sexes. Sometimes fitness of only one sex matters, that is, either viability of females or the product of viability and fertility in males (which is their lifetime reproductive success). Once the sex-determining factor does invade, however, the final state of the system (full switch to different sex-determining system) is usually independent of whether male or female fitness is affected (at least for the numerical scenarios considered by us). Therefore, our assumptions on the fertility or viability effects of segregation distorters do not seem to substantially affect our predictions. The situation may be different if the population is structured into small local mating groups or when different mating patterns occur (Van Boven and Weissing, 1999; Pen, 2006).

In some of our simulations, a complete switch to a new sex-determining system could only be achieved if the standard $X$ or $Y$ chromosome was reintroduced in the population. This is more plausible than it may appear at first sight. In our model, the standard $X$ or $Y$ chromosome was completely driven out of the population. However, in natural systems, a population often consists of many subpopulations with limited gene flow between them. Therefore, one can easily imagine that a driving element will initially appear only in one subpopulation and spread within it, and subsequently to other populations. However, if a new sex-determining factor appears before the whole population is fixed for a driving allele, migration of standard chromosomes from other subpopulations will lead to spread of new sex-determining factor even in subpopulations in which the driving element is fixed. Alternatively, even if the driving allele is fixated in the whole population, any mutation restoring vitality or fertility of homozygotes will be favoured and spread in the population, even if it does not have a segregation advantage.

Perhaps the most interesting result of our simulations is that the segregation distorter, whose presence initiated the change in the sex-determination system, is often subsequently lost from the population. Perhaps surprisingly, such an effect has never been found in previous models (Bengtsson, 1977; Jayakar, 1987; Haig, 1993a, b; McVean and Hurst, 1996; Charlesworth and Dempsey, 2001). In these models, a change in the sex-determining system usually neutralizes drive, for example, by removing sensitive alleles, but never leads to the complete loss of the driving allele. However, our results show that the presence of a segregation distorter can only be a transient state, the traces of which are no longer visible once a new sex-determination system has become established.

Loss of the driver would make it difficult to detect the role of segregation distortion in the evolution of extant sex-determining mechanisms, especially given that changes to a new sex-determining mechanism are often very rapid (Figures 1-3). Indication of the role of segregation distortion in the evolution of sex-determining mechanisms could be found indirectly, for example, by looking at closely related species of a species in which sex chromosome meiotic drive has been found, to check whether change in sex determination occurred and whether they are consistent with our results (for example, if $X$ drives, invasion of a new male-determining factor is expected). Alternatively, in a species or a closely related group of species in which sex-determining genes are known to be located on different chromosomes (for example, medaka fish, Takehana et al., 2007; the fly Megasela scalaris, Traut and Willhoeft, 1990), one could investigate whether some of the populations (species) 
possess driving sex chromosomes. If in such populations, the sex-determining mechanism is ancestral to other populations, this could suggest that segregation distortion was responsible for the change in sex-determining mechanisms. However, this is only circumstantial evidence.

A more direct test of the predictions of our model would probably demand laboratory experiments. They could be set up using (closely related) species in which multiple sex-determining factors exist together with segregation distorters. The housefly, Musca domestica, seems to be an especially good candidate. In this species, a variety of sex-determining mechanisms coexist in natural populations, such as a standard XY system, as well as autosomal male and female-determining factors, with effects on sex determination, as described in our model (Dübendorfer et al., 2002). In addition, in some North American populations, autosomal $M$ factors show segregation distortion of 0.75-0.9 (Clark, 1999; although driving $M^{\prime}$ s are probably absent in European populations; Kozielska, 2008). Unfortunately, changes in the frequency of sex-determining factors in these North American populations have not been studied. Even though segregation distortion is probably not the main driving force for the evolution of sex determination in natural populations of the housefly (Feldmeyer et al., 2008; Kozielska et al., 2008), experiments could be set up to test predictions of our model. In addition, one could study frequencies of different factors in natural populations with segregation distortion over long periods of time. In addition, in other species with multiple sexdetermining factors, segregation distortion could be induced with molecular techniques (Windbichler et al., 2008) and subsequent dynamics of sex-determining mechanisms could be followed.

In summary, our simulations show that in many cases segregation distortion creates selective pressure allowing novel sex-determining alleles to spread and induce shifts to a new sex-determining system. Interestingly, the segregation distorter, the presence of which initiated this shift, can be subsequently lost from the population. Therefore, the role of segregation distortion in the evolution of sex-determining mechanisms may be underestimated.

\section{References}

Atlan A, Capillon C, Derome N, Couvet D, Montchamp-Moreau C (2003). The evolution of autosomal suppressors of sex-ratio drive in Drosophila simulans. Genetica 117: 47-58.

Atlan A, Joly D, Capillon C, Montchamp-Moreau C (2004). Sexratio distorter of Drosophila simulans reduces male productivity and sperm competition ability. J Evol Biol 17: 744-751.

Bengtsson BO (1977). Evolution of the sex ratio in the wood lemming, Myopus schisticolor. In: Christiansen FB, Fenchel TM (eds). Measuring Selection in Natural Populations. Springer: Berlin, pp 333-343.

Buckler ES, Phelps-Durr TL, Keith Buckler CS, Dawe RK, Doebley JF, Holtsford TP (1999). Meiotic drive of chromosomal knobs reshaped the maize genome. Genetics 153: 415-426.

Bull JJ (1983). The Evolution of Sex Determining Mechanisms. Benjamin/Cummings Publishing Co: Menlo Park, CA.

Bull JJ, Charnov EL (1977). Changes in the heterogametic mechanism of sex determination. Heredity 39: 1-14.

Burt A, Trivers R (2006). Genes in Conflict. The Belknap Press of Harvard University Press: Cambridge, Massachusetts/ London, England.
Carvalho de AB, Peixoto AA, Klaczko LB (1989). Sex-ratio in Drosophila mediopunctata. Heredity 62: 425-428.

Charlesworth B, Dempsey ND (2001). A model of the evolution of the unusual sex chromosome system of Microtus oregoni. Heredity 86: 387-394.

Charlesworth D, Charlesworth B, Marais G (2005). Steps in the evolution of heteromorphic sex chromosomes. Heredity 95: 118-128.

Clark ME (1999). The evolution of a neo-Y chromosome in the housefly, Musca domestica. PhD Thesis. University of Houston, TX, USA.

Cosmides LM, Tooby J (1981). Cytoplasmic inheritance and intragenomic conflict. J Theor Biol 89: 83-129.

Curtsinger JW, Feldman MW (1980). Experimental and theoretical analysis of the sex-ratio polymorphism in Drosophila pseudoobscura. Genetics 94: 445-466.

Dübendorfer A, Hediger M, Burghardt G, Bopp D (2002). Musca domestica, a window on the evolution of sex-determining mechanisms in insects. Int J Dev Biol 46: 75-79.

Feldmeyer B, Kozielska M, Weissing FJ, Beukeboom LW, Pen I (2008). Temperature and the geographical distribution of sex determining mechanisms in the housefly. Evol Ecol Res 10: 797-809.

Fraser JA, Heitman J (2005). Chromosomal sex-determining regions in animals, plants and fungi. Curr Opin Genet Dev 15: 645-651.

Gileva EA (1987). Meiotic drive in the sex chromosome system of the varying lemming, Dicrostonyx torquatus Pall. (Rodentia, Microtinae). Heredity 59: 383-389.

Haig D (1993a). The evolution of unusual chromosomal systems in coccoids-extraordinary sex ratios revisited. J Evol Biol 6: 69-77.

Haig D (1993b). The evolution of unusual chromosomal systems in sciarid flies-intragenomic conflict and the sex ratio. J Evol Biol 6: 249-261.

Hamilton WD (1967). Extraordinary sex ratios. Science 156: 477-488.

Hickey WA, Craig GB (1966). Genetic distortion of sex ratio in a mosquito Aedes aegypti. Genetics 53: 1177-1196.

Hiroyoshi T (1977). Some new mutants and revised linkage maps of housefly, Musca domestica L. Jpn J Genet 52: 275-288.

Hurst LD, Atlan A, Bengtsson BO (1996). Genetic conflicts. Quart Rev Biol 71: 317-364.

Jaenike J (1996). Sex-ratio meiotic drive in the Drosophila quinaria group. Am Nat 148: 237-254.

Jaenike J (1999). Suppression of sex-ratio meiotic drive and the maintenance of Y-chromosome polymorphism in Drosophila. Evolution 53: 164-174.

Jaenike J (2001). Sex chromosome meiotic drive. Annu Rev Ecol Syst 32: 25-49.

Janzen FJ, Phillips PC (2006). Exploring the evolution of environmental sex determination, especially in reptiles. J Evol Biol 19: 1775-1784.

Jayakar SD (1987). Some two locus models for the evolution of sex-determining mechanisms. Theor Pop Biol 32: 188-215.

Juchault P, Rigaud T (1995). Evidence for female heterogamety in two terrestrial crustaceans and the problem of sex chromosome evolution in isopods. Heredity 75: 466-471.

Kozielska M, Feldmeyer B, Pen I, Weissing FJ, Beukeboom LW (2008). Are autosomal sex determining factors of the housefly (Musca domestica) spreading north? Genet Res 90: 157-165.

Kozielska M, Pen I, Beukeboom LW, Weissing FJ (2006). Sex ratio selection and multi-factorial sex determination in the housefly: a dynamic model. I Evol Biol 19: 879-888.

Kozielska MA (2008). Evolutionary dynamics of sex determination. Mechanistic theory and empirical investigations. $\mathrm{PhD}$ Thesis. University of Groningen, Groningen, The Netherlands. http://irs.ub.rug.nl/ppn/310130085.

Kraak SBM, Pen I (2002). Sex-determining mechanisms in vertebrates. In: Hardy ICW (ed). Sex Ratios Concepts and 
Research Methods. Cambridge University Press: Cambridge, pp 158-177.

Lyttle TW (1981). Experimental population genetics of meiotic drive systems. III. Neutralization of sex ratio distortion in Drosophila through sex chromosome aneuploidy. Genetics 98: 317-334.

Lyttle TW (1991). Segregation distorters. Annu Rev Genet 25: 511-557.

Lyttle TW (1993). Cheaters sometimes prosper-distortion of Mendelian segregation by meiotic drive. Trends Genet 9: 205-210.

Martin J, Kuvangkadilok C, Peart DH, Lee BTO (1980). Multiple sex determining regions in a group of related Chironomus species (Diptera, Chironomidae). Heredity 44: 367-382.

McVean G, Hurst LD (1996). Genetic conflicts and the paradox of sex determination: Three paths to the evolution of female intersexuality in a mammal. J Theor Biol 179: 199-211.

Montchamp-Moreau C, Ginhoux V, Atlan A (2001). The Y chromosomes of Drosophila simulans are highly polymorphic for their ability to suppress sex-ratio drive. Evolution 55: 728-737.

Otto SP, Day T (2007). A Biologist's Guide to Mathematical Modeling in Ecology and Evolution. Princeton University Press: Princeton and Oxford.

Pen I (2006). When boys want to be girls: effects of mating system and dispersal on parent-offspring sex ratio conflict. Evol Ecol Res 8: 103-113.

Smith DAS, Gordon IJ, Depew LA, Owen DF (1998). Genetics of the butterfly Danaus chrysippus (L.) in a broad hybrid zone, with special reference to sex ratio, polymorphism and intergenomic conflict. Biol J Linn Soc 65: 1-40.

Sturtevant AH, Dobzhansky T (1936). Geographical distribution and cytology of 'sex ratio' in Drosophile pseudoobscura and related species. Genetics 21: 473-490.

Takehana Y, Demiyah D, Naruse K, Hamaguchi S, Sakaizumi M (2007). Evolution of different $Y$ chromosomes in two medaka species, Oryzias dancena and $O$ latipes. Genetics 175: 1335-1340.

Taylor DR, Ingvarsson PK (2003). Common features of segregation distortion in plants and animals. Genetica 117: 27-35.

Traut W, Willhoeft U (1990). A jumping sex determining factor in the fly Megaselia scalaris. Chromosoma 99: 407-412.

Uller T, Pen I, Wapstra E, Beukeboom LW, Komdeur J (2007). The evolution of sex ratios and sex-determining systems. Trends Ecol Evol 22: 292-297.

Van Boven M, Weissing FJ (1998). Evolution of segregation distortion: potential for a high degree of polymorphism. J Theor Biol 192: 131-142.

Van Boven M, Weissing FJ (1999). Segregation distortion in a deme structured population: opposing demands of gene, individual and group selection. J Evol Biol 12: 80-93.

Wallace B (1948). Studies on 'sex ratio' in Drosophila pseudoobscura .1. Selection and 'sex ratio'. Evolution 2: 189-217.

Werren JH, Beukeboom LW (1998). Sex determination, sex ratios, and genetic conflict. Annu Rev Ecol Syst 29: 233-261.

Wilkinson GS, Johns PM, Kelleher ES, Muscedere ML, Lorsong A (2006). Fitness effects of $X$ chromosome drive in the stalk-eyed fly, Cyrtodiopsis dalmanni. J Evol Biol 19: 1851-1860.

Windbichler N, Papathanos PA, Crisanti A (2008). Targeting the $\mathrm{X}$ chromosome during spermatogenesis induces $\mathrm{Y}$ chromosome transmission ratio distortion and early dominant embryo lethality in Anapheles gambiae. PLoS Genetics 4: e1000291 (doi:10.1371/journal.pgen.1000291).

\section{Appendix A}

We model the evolutionary dynamics of the sexdetermining system with a set of recurrence equations similar to the one described by us earlier (Kozielska et al., 2006), although with slightly different notation. We consider three independent sex-determining loci and we encode genotypes by triplets $\mathbf{i}=\left(\mathbf{i}_{1}, \mathbf{i}_{2}, \mathbf{i}_{3}\right)$, where $\mathbf{i}_{n}$ corresponds to the genotype at locus $n$. At each locus, the genotypes are unordered, meaning that the heterozygous genotype $A B$ is equivalent to $B A$. The sexual phenotype determined by genotype $\mathbf{i}$ is encoded as a binary variable: $s_{\mathbf{i}}=0$ for females and $s_{\mathbf{i}}=1$ for males. The frequencies of genotype $\mathbf{i}$ among adult females and males are written as $x_{\mathbf{i}}$ and $y_{\mathbf{i}}$, respectively $\left(\sum_{\mathbf{i}} x_{\mathbf{i}}=\sum_{\mathbf{i}} y_{\mathbf{i}}=1\right)$. Note that for each $\mathbf{i}$, either $x_{\mathbf{i}}=0$ or $y_{\mathbf{i}}=0$, because the genotype $\mathbf{i}$ uniquely determines sex.

\section{Fitness}

Each genotype has its specific viability $v$ and fertility $u$. In our model, all females have the same fertility, hence $u_{f, \mathbf{i}}=1$. Male fertility may depend on genotype and is denoted by $u_{m, \mathrm{i}}$. For numerical examples in the main text, we considered three fitness schemes: (a) no cost of homozygosity for the driving allele, that is, $v_{\mathbf{i}}=u_{m, \mathbf{i}}=1$ for all $\mathbf{i}$; (b) males homozygous for a driving allele are completely sterile, that is, $u_{m, \mathbf{i}}=0$ when $\mathbf{i}$ is homozygous for a driving allele and $u_{m, \mathbf{i}}=1$ otherwise; (c) homozygosity for a driving allele is lethal in both sexes, that is, $v_{\mathbf{i}}=0$ if $\mathbf{i}$ is homozygous for a driving allele and $v_{\mathbf{i}}=1$ otherwise.

\section{Transmission}

The frequency of genotype $\mathbf{z}$ among the offspring of a cross between an $\mathbf{i}$-female and a $\mathbf{j}$-male is denoted by $T_{\mathbf{z}, \mathbf{i j}}$, which can be decomposed into three per locus components:

$$
T_{\mathbf{z}, \mathbf{i j}}=P\left(\mathbf{z}_{1} \mid \mathbf{i}_{1} \mathbf{j}_{1}\right) P\left(\mathbf{z}_{2} \mid \mathbf{i}_{2} \mathbf{j}_{2}\right) P\left(\mathbf{z}_{3} \mid \mathbf{i}_{3} \mathbf{j}_{3}\right),
$$

where $P\left(\mathbf{z}_{n} \mid \mathbf{i}_{n} \mathbf{j}_{n}\right)$ is the probability that at locus $n$, the offspring genotype will be $\mathbf{z}_{n}$ given a maternal genotype $\mathbf{i}_{n}$ and paternal genotype $\mathbf{j}_{n}$.

If offspring genotype at locus $n$ is homozygous for allele $A$, then

$$
P\left(\mathbf{z}_{n}=A A \mid \mathbf{i}_{n} \mathbf{j}_{n}\right)=\frac{1}{2} q_{A, \mathbf{i}_{n}} k_{A, \mathbf{j}_{n}} q_{A, \mathbf{j}_{n}},
$$

where $q_{A, \mathbf{i}_{n}}$ and $q_{A, \mathbf{j}_{n}}$ are the frequencies of $A$ alleles in mother and father, and $k_{A, \mathbf{j}_{n}}$ is the segregation ratio (proportion of gametes) of the $A$ allele in $\mathbf{j}_{n}$-males. If $\mathbf{j}_{n}$ is heterozygous for a driving allele $A$ and a non-driving allele $a$, then $k_{A, \mathbf{j}_{n}}=k$ and $k_{a, \mathbf{j}_{n}}=1-k$, otherwise $k_{A, \mathbf{j}_{n}}=\frac{1}{2}$ ('honest segregation').

For heterozygous offspring with genotype $A B$ at locus $n$ :

$$
P\left(\mathbf{z}_{n}=A B \mid \mathbf{i}_{n} \mathbf{j}_{n}\right)=\frac{1}{2} q_{A, \mathbf{i}_{n}} k_{B, \mathbf{j}_{n}} q_{B, \mathbf{j}_{n}}+\frac{1}{2} q_{B, \mathbf{i}_{n}} k_{A, \mathbf{j}_{n}} q_{A, \mathbf{j}_{n}} .
$$

\section{Recurrence equations}

Under random mating, a random mating pair consists of an $\mathbf{i}$-female and a $\mathbf{j}$-male with probability given by the product of their frequencies, that is, $x_{\mathbf{i}} y_{\mathrm{j}}$. Allowing for fertility differences between males, the frequency of z-offspring before viability selection equals

$$
\bar{T}_{\mathbf{z}}=\frac{1}{\bar{u}_{m}} \sum_{\mathbf{i j}} x_{\mathbf{i}} y_{\mathbf{j}} u_{m, \mathbf{j}} T_{\mathbf{z}, \mathbf{i} \mathbf{j}}
$$

where $\bar{u}_{m}=\sum_{\mathbf{j}} y_{\mathbf{j}} u_{m, \mathbf{j}}$ is the mean fertility of males.

Assuming discrete and non-overlapping generations, the genotype frequencies after one round of reproduction 
and selection are given by

$$
x_{\mathbf{z}}^{\prime}=\frac{v_{\mathbf{z}}\left(1-s_{\mathbf{z}}\right) \bar{T}_{\mathbf{z}}}{\bar{v}(1-S)}, y_{\mathbf{z}}^{\prime}=\frac{v_{\mathbf{z}} s_{\mathbf{z}} \bar{T}_{\mathbf{z}}}{\bar{v} S},
$$

where $\bar{v}=\sum_{z} v_{z} \bar{T}_{z}$ is mean offspring viability and $S=(1 / \bar{v}) \sum_{\mathbf{z}} v_{\mathbf{z}} \bar{T}_{\mathbf{z}} s_{\mathbf{z}}$ is the sex ratio (proportion males) after viability selection.

\section{Appendix B}

To support and generalize the numerical analysis in the main text, we here perform an invasion analysis for driving alleles and new sex-determining factors. We follow the same sequence of scenarios, as in the main text. Scenario 1: driving $X$ chromosome

We work with a two-locus model, with three alleles $(X, Y$, $\left.X^{d}\right)$ at the $X Y$ locus and two alleles $(m, M)$ at the unlinked autosomal $\mathrm{M}$ locus. Therefore, there are three female genotypes (XXmm, $X^{\mathrm{d}} X m m$ and $\left.X^{\mathrm{d}} X^{\mathrm{d}} m m\right)$ and seven male genotypes (XYmm, $X^{\mathrm{d} Y m m}, X^{\mathrm{d}} Y M m, X^{\mathrm{d}} X^{\mathrm{d}} M m, X Y M m$, $X X M m$ and $\left.X^{\mathrm{d}} X M m\right)$, with frequencies $x_{1} \ldots x_{3}$ and $y_{1} \ldots y_{7}$, respectively. When paired with a $\mathrm{Y}$ chromosome in males, the driving allele $X^{\mathrm{d}}$ has a segregation ratio of $k>\frac{1}{2}$, otherwise segregation is 'honest'. We assume that males homozygous for $X^{\mathrm{d}}$ have relative fertility $0 \leqslant u \leqslant 1$ (males are sterile when $u=0$ ) and viability $0 \leqslant v_{m} \leqslant 1$, and females homozygous for $X^{\mathrm{d}}$ have viability $v_{f}$. Then the full dynamics is given by the recursions (4):

$$
\begin{aligned}
C_{\mathrm{f}} x_{1}^{\prime}= & y_{1}\left(\frac{1}{2} x_{1}+\frac{1}{4} x_{2}\right)+y_{5}\left(\frac{1}{4} x_{1}+\frac{1}{8} x_{2}\right) \\
& +y_{6}\left(\frac{1}{2} x_{1}+\frac{1}{4} x_{2}\right)+y_{7}\left(\frac{1}{4} x_{1}+\frac{1}{8} x_{2}\right) \\
C_{\mathrm{f}} x_{2}^{\prime}= & y_{1}\left(\frac{1}{4} x_{2}+\frac{1}{2} x_{3}\right)+k y_{2}\left(x_{1}+\frac{1}{2} x_{2}\right) \\
& +k y_{3}\left(\frac{1}{2} x_{1}+\frac{1}{4} x_{2}\right)+u y_{4}\left(\frac{1}{2} x_{1}+\frac{1}{4} x_{2}\right) \\
& +y_{5}\left(\frac{1}{8} x_{2}+\frac{1}{4} x_{3}\right)+y_{6}\left(\frac{1}{4} x_{2}+\frac{1}{2} x_{3}\right)+\frac{1}{4} y_{7} \\
C_{\mathrm{f}} x_{3}^{\prime}= & v_{f}\left[k y_{2}\left(\frac{1}{2} x_{2}+x_{3}\right)+k y_{3}\left(\frac{1}{4} x_{2}+\frac{1}{2} x_{3}\right)\right. \\
& \left.+u y_{4}\left(x_{2}+\frac{1}{2} x_{3}\right)+y_{7}\left(\frac{1}{8} x_{2}+\frac{1}{4} x_{3}\right)\right] \\
C_{\mathrm{m}} y_{1}^{\prime}= & y_{1}\left(\frac{1}{2} x_{1}+\frac{1}{4} x_{2}\right)+(1-k) y_{2}\left(x_{1}+\frac{1}{2} x_{2}\right) \\
& +(1-k) y_{3}\left(\frac{1}{2} x_{1}+\frac{1}{4} x_{2}\right)+y_{5}\left(\frac{1}{4} x_{1}+\frac{1}{8} x_{2}\right) \\
C_{\mathrm{m}} y_{2}^{\prime}= & y_{1}\left(\frac{1}{4} x_{2}+\frac{1}{2} x_{3}\right)+(1-k) y_{2}\left(\frac{1}{2} x_{2}+x_{3}\right) \\
& +(1-k) y_{3}\left(\frac{1}{4} x_{2}+\frac{1}{2} x_{3}\right)+y_{5}\left(\frac{1}{8} x_{2}+\frac{1}{4} x_{3}\right) \\
C_{\mathrm{m}} y_{3}^{\prime}= & (1-k) y_{3}\left(\frac{1}{4} x_{2}+\frac{1}{2} x_{3}\right)+y_{5}\left(\frac{1}{8} x_{2}+\frac{1}{4} x_{3}\right) \\
C_{\mathrm{m}} y_{4}^{\prime}= & v_{m}\left[k y_{3}\left(\frac{1}{4} x_{2}+\frac{1}{2} x_{3}\right)+u y_{4}\left(\frac{1}{4} x_{2}+\frac{1}{2} x_{3}\right)\right. \\
& \left.+y_{7}\left(\frac{1}{8} x_{2}+\frac{1}{4} x_{3}\right)\right] \\
C_{\mathrm{m}} y_{5}^{\prime}= & (1-k) y_{3}\left(\frac{1}{2} x_{1}+\frac{1}{4} x_{2}\right)+y_{5}\left(\frac{1}{4} x_{1}+\frac{1}{8} x_{2}\right) \\
C_{\mathrm{m}} y_{6}^{\prime}= & y_{5}\left(\frac{1}{4} x_{1}+\frac{1}{8} x_{2}\right)+y_{6}\left(\frac{1}{2} x_{1}+\frac{1}{4} x_{2}\right) \\
& +y_{7}\left(\frac{1}{4} x_{1}+\frac{1}{8} x_{2}\right) \\
C_{\mathrm{m}} y_{7}^{\prime}= & k y_{3}\left(\frac{1}{2} x_{1}+\frac{1}{4} x_{2}\right)+u y_{4}\left(\frac{1}{2} x_{1}+\frac{1}{4} x_{2}\right) \\
& +y_{5}\left(\frac{1}{8} x_{2}+\frac{1}{4} x_{3}\right)+y_{6}\left(\frac{1}{4} x_{2}+\frac{1}{2} x_{3}\right)+\frac{1}{4} y_{7}
\end{aligned}
$$

Here $C_{\mathrm{f}}=\bar{u}_{m} \bar{v}(1-S)$ and $C_{\mathrm{m}}=\bar{u}_{m} \bar{v} S$ are normalization factors, as defined in Appendix A.

Invasion of $X^{\mathrm{d}}$ into the standard $\mathrm{XY}$ system: To determine the stability of the $X Y$ system with respect to invasion of rare $X^{\mathrm{d}}$ chromosomes, we calculated the Jacobian of system (B1), restricted to the subspace without the $M$ allele, evaluated at the equilibrium point

$$
\begin{aligned}
\hat{\mathbf{z}}_{X Y} & =\left(\hat{\mathbf{x}}_{1}=1, \ldots, \mathbf{y}_{1}=1, \ldots\right): \\
J & =\left[\begin{array}{lllll}
\frac{\partial x_{1}^{\prime}}{\partial x_{1}} & \frac{\partial x_{1}^{\prime}}{\partial x_{2}} & \frac{\partial x_{1}^{\prime}}{\partial x_{3}} & \frac{\partial x_{1}^{\prime}}{\partial y_{1}} & \frac{\partial x_{1}^{\prime}}{\partial y_{2}} \\
\frac{\partial x_{2}^{\prime}}{\partial x_{1}} & \frac{\partial x_{2}^{\prime}}{\partial x_{2}} & \frac{\partial x_{2}^{\prime}}{\partial x_{3}} & \frac{\partial x_{2}^{\prime}}{\partial y_{1}} & \frac{\partial x_{2}^{\prime}}{\partial y_{2}} \\
\frac{\partial x_{3}^{\prime}}{\partial x_{1}} & \frac{\partial x_{3}^{\prime}}{\partial x_{2}} & \frac{\partial x_{3}^{\prime}}{\partial x_{3}} & \frac{\partial x_{3}^{\prime}}{\partial y_{1}} & \frac{\partial x_{3}^{\prime}}{\partial y_{2}} \\
\frac{\partial y_{1}^{\prime}}{\partial x_{1}} & \frac{\partial y_{1}^{\prime}}{\partial x_{2}} & \frac{\partial y_{1}^{\prime}}{\partial x_{3}} & \frac{\partial y_{1}^{\prime}}{\partial y_{1}} & \frac{\partial y_{1}^{\prime}}{\partial y_{2}} \\
\frac{\partial y_{2}^{\prime}}{\partial x_{1}} & \frac{\partial y_{2}^{\prime}}{\partial x_{2}} & \frac{\partial y_{2}^{\prime}}{\partial x_{3}} & \frac{\partial y_{2}^{\prime}}{\partial y_{1}} & \frac{\partial y_{2}^{\prime}}{\partial y_{2}}
\end{array}\right] \\
& =\left[\begin{array}{ccccc}
0 & -\frac{1}{2} & -1 & 0 & -2 k \\
0 & \frac{1}{2} & 1 & 0 & 2 k \\
0 & 0 & 0 & 0 & 0 \\
0 & -\frac{1}{2} & -1 & 0 & 0 \\
0 & \frac{1}{2} & 1 & 0 & 0
\end{array}\right]
\end{aligned}
$$

A rare $X^{\mathrm{d}}$ chromosome will spread if the leading eigenvalue of $J$ is larger than 1 (for example, Otto and Day, 2007). In this case, the leading eigenvalue of $J$ is $\lambda_{1}=\frac{1}{4}(1+\sqrt{1+16 k})$. As $\lambda_{1}>1$ if and only if $k>\frac{1}{2}$, a rare driving $X^{\mathrm{d}}$ chromosome can always invade an $X Y$ population, regardless of its effect on viability and male fertility when in homozygous condition.

With $X^{\mathrm{d}}$ present and $M$ (still) absent, there are at most two equilibria in addition to the unstable equilibrium $\hat{\mathbf{z}}_{X Y}$. One of these equilibria corresponds to fixation of $X^{\mathrm{d}}\left(\hat{x}_{3}=1, \hat{y}_{2}=1\right)$. If female viability, $v_{f}$, is sufficiently large, this is the only equilibrium. For $v_{f}<\frac{1}{2}+\frac{1}{4 k}$ there is an additional equilibrium in which $X$ and $X^{\mathrm{d}}$ coexist. Specifically, in the sterility scenario of Table $2(u=0$, $\left.v_{f}=v_{m}=1\right)$, there is only an equilibrium with $X^{\mathrm{d}}$ fixed and an adult sex ratio given by $\hat{S}=1-k$, whereas in the lethality scenario $\left(u=1, v_{f}=v_{m}=0\right)$, there is only a polymorphic equilibrium $\left(\hat{z}_{1}=\frac{1}{2 k}, \hat{z}_{2}=1-\frac{1}{2 k}, \hat{y}_{1}=\frac{1}{2}+\frac{1}{4 k}, \hat{y}_{2}=\frac{1}{2}-\frac{1}{4 k}\right)$ and the adult sex ratio is bounded by $\frac{2}{5}$ from below (when $k=1$ ) and by $\frac{1}{2}$ from above $\left(k=\frac{1}{2}\right)$. If a polymorphic equilibrium exists, it is always locally stable and the fixation equilibrium is then unstable against invasion by $X$, while the fixation equilibrium is stable against invasion by $X$ if there is no polymorphic equilibrium.

Invasion of $M$ : Stability against invasion of $M$ of the equilibrium with $X^{\mathrm{d}}$ fixed is determined by the Jacobian restricted to the subspace without the $X$ allele, evaluated at $\hat{\mathbf{z}}_{X^{\mathrm{d}}}=\left(\hat{x}_{3}=1, \hat{y}_{2}=1\right)$ :

$$
J=\left[\begin{array}{cccc}
0 & 0 & 0 & 0 \\
0 & 0 & -\frac{1-k\left(1-v_{m}\right)}{2(1-k)} & -\frac{v_{m} u}{2(1-k)} \\
0 & 0 & 1 / 2 k & 0 \\
0 & 0 & \frac{v_{m} k}{2(1-k)} & \frac{v_{m} u}{2(1-k)}
\end{array}\right]
$$

The leading eigenvalue of $J$ is either $\lambda_{1}=\frac{1}{2} v_{m} u /(1-k)$ or $\lambda_{2}=\frac{1}{2}$. Thus, if the driving allele in homozygous condition confers complete male sterility $(u=0)$ or complete male lethality $\left(v_{m}=0\right)$, the $M$ allele cannot invade a population with $X^{\text {d }}$ fixed. Invasion will occur only if $k>1-\frac{1}{2} v_{m} u$, in other words, as long as $X^{\mathrm{d}}$ does not cause complete sterility or inviability, $M$ can invade provided $k$ is sufficiently large.

It is difficult to analyse invasion stability of the polymorphic equilibrium against invasion of $M$ in full 
generality. For the special case of lethality $\left(v_{f}=v_{m}=0\right)$, the leading eigenvalue of the $10 \times 10$ jacobian is independent of $u$ and given by

$$
\lambda_{1}=\frac{1+4 k+\sqrt{8 k^{2}-1}}{2\left(8 k-1-4 k^{2}\right)}>1 \Leftrightarrow k>\frac{1}{2}
$$

Hence $M$ can always invade. In the general case, we can approximate the leading eigenvalue near $\varepsilon=k-\frac{1}{2}$ :

$$
\lambda_{1}=1+\frac{1+v_{m} u-2 v_{f}}{\left(1-v_{f}\right)^{2}} \varepsilon^{2}+O\left(\varepsilon^{3}\right)
$$

It can be seen that this expression may be smaller than unity, hence $M$ cannot always invade. In particular, if $2 v_{f}>1+v_{m} u$, then $M$ cannot invade for sufficiently small $k$.

Simultaneous invasion of $M$ and $X$ when $X^{\mathrm{d}}$ is fixed: The situation is different when $X$ and $M$ are simultaneously introduced at low frequency in a population otherwise fixed for $X^{\mathrm{d}}$. Then the relevant Jacobian matrix is given by

$$
J=\left[\begin{array}{ccccc}
0 & 0 & 0 & 0 & 0 \\
\frac{1}{v_{f}} & \frac{1}{2 v_{f}} & 0 & \frac{1}{2 k v_{f}} & 0 \\
-\frac{1}{v_{f}} & -\frac{1}{2 v_{f}} & 0 & -\frac{1}{2 k v_{f}} & 0 \\
1 & \frac{1}{2} & 0 & 0 & 0 \\
-1 & -\frac{1}{2} & 0 & 0 & 0 \\
0 & 0 & 0 & 0 & 0 \\
0 & 0 & 0 & 0 & 0 \\
0 & 0 & 0 & 0 & 0 \\
0 & 0 & 0 & 0 & 0 \\
0 & 0 & 0 & 0 & 0
\end{array}\right.
$$$$
\begin{gathered}
0 \\
0 \\
0 \\
0 \\
-\frac{1-k\left(1-v_{m}\right)}{2(1-k)} \\
\frac{1}{2} \\
\frac{k v_{m}}{2(1-k)} \\
0 \\
0 \\
0
\end{gathered}
$$$$
\begin{gathered}
0 \\
0 \\
0 \\
0 \\
\frac{v_{m} u}{2(1-k)} \\
0 \\
\frac{v_{m} u}{2(1-k)} \\
0 \\
0 \\
0
\end{gathered}
$$

0
$\frac{1}{4 k v_{f}}$
$-\frac{1}{4 k v_{f}}$
0
$\frac{1}{2(1-k)}$
$\frac{1}{4(1-k)}$
0
0
0
$\frac{1}{4(1-k)}$

$\left.\begin{array}{cc}0 & 0 \\ \frac{1}{2 k v_{f}} & \frac{1}{4 k v_{f}} \\ -\frac{1}{2 k v_{f}} & -\frac{1}{4 k v_{f}} \\ 0 & 0 \\ \frac{1}{2(1-k)} & -\frac{1+v_{m}}{4(1-k)} \\ 0 & 0 \\ 0 & \frac{v_{m}}{4(1-k)} \\ 0 & 0 \\ 0 & 0 \\ \frac{1}{2(1-k)} & \frac{1}{4(1-k)}\end{array}\right]$

Three candidate leading eigenvalues are

$$
\begin{aligned}
& \lambda_{1}=\frac{k+\sqrt{k\left(k+4 v_{f}\right)}}{4 k v_{f}} \\
& \lambda_{2}=\frac{v_{m} u}{2(1-k)} \\
& \lambda_{3}=\frac{1}{4(1-k)}
\end{aligned}
$$

Since $\lambda_{3}>1 \Leftrightarrow k>\frac{3}{4}$, the equilibrium with $X^{\mathrm{d}}$ fixed is always unstable against simultaneous invasion of $X$ and $M$ whenever $k>\frac{3}{4}$. In case of complete male sterility $(u=0)$ and full female viability $\left(v_{f}=1\right)$ of $X^{\mathrm{d}} X^{\mathrm{d}}$ genotypes, $\lambda_{1}>1 \Leftrightarrow k>\frac{1}{2}$ and $\lambda_{2}=0$, hence in this case, $k>\frac{3}{4}$ is necessary and sufficient for invasion of $M$ (Table 2). In addition note that for sufficiently small $v_{f}, \lambda_{1}>1$ for any $k>\frac{1}{2}$. Specifically, if $X^{\mathrm{d}} X^{\mathrm{d}}$ females are lethal, then $M$ can always invade together with $X$.

\section{Scenario 2: driving $Y$ chromosome}

We study a two-locus system with seven female genotypes (XXff, XXFf, XYFf, YYFf, XY ${ }^{\mathrm{d}} F f, Y Y^{\mathrm{a}} F f$ and $\left.Y^{\mathrm{d}} Y^{\mathrm{d}} F f\right)$ with frequencies $x_{1} \ldots x_{7}$ and five male genotypes $\left(X Y f f, Y Y f f, X Y^{\mathrm{d} f f}, Y Y^{\mathrm{d}} f f\right.$ and $\left.Y^{\mathrm{d}} Y^{\mathrm{d}} f f\right)$ with frequencies $y_{1} \ldots y_{5}$. An $Y^{\mathrm{d}}$ chromosome only drives when paired with an $X$ chromosome. Males homozygous for $Y^{\mathrm{d}}$ have fertility $0 \leqslant u \leqslant 1$ and viability $0 \leqslant v_{m} \leqslant 1$, whereas females homozygous for $Y^{\mathrm{d}}$ have viability $0 \leqslant v_{f} \leqslant 1$. The twelve recursion equations are too bulky to reproduce here in full. A Maple (version 11) code containing the algebra is available upon request from the authors.

Invasion of $Y^{\mathrm{d}}$ into the standard $X Y$ system: The relevant Jacobian matrix, restricted to the subspace without $F$ and evaluated at the $\mathrm{XY}$ equilibrium has leading eigenvalue $2 k$, regardless of the values $u, v_{m}$ and $v_{f}$. Hence $Y^{d}$ can always invade provided $k>\frac{1}{2}$. The equilibrium in which $Y$ is lost and all males are $X Y^{\mathrm{d}}$ is stable.

Invasion of $F$ into the $X Y^{\mathrm{a} / f f}$ system: The relevant $12 \times 12$ Jacobian matrix has leading eigenvalue

$$
\lambda_{1}=\frac{3-2 k\left(1-v_{f}\right)+\sqrt{1+4 k\left(1-k-v_{f}\left(1-v_{f}\right)\right)}}{8(1-k)}
$$

Hence $\lambda_{1}$ is independent of $u$ and $v_{m}$, and increases with $v_{f}$. For full viability of homozygous females $\left(v_{f}=1\right)$, 
It can be shown that $\lambda_{1}>1 \Leftrightarrow k>\frac{1}{2}$, hence $Y$ can always invade when $u=0$ and $v_{f}=1$ (Table 3 ).

For the scenario with lethality $\left(u=1, v_{f}=v_{m}=0\right)$, analytical results are not insightful. Numerical calculations of eigenvalues show that $Y$ can invade when $k>0.655$.

\section{Scenario 3: driving $M^{\mathrm{d}}$}

Now, we work with a three-locus system with 10 female genotypes (XXmmff, XXmmFf, XYmmFf, YYmmFf, $X X m M^{\mathrm{d}} F f, X Y m M^{\mathrm{d}} F f, Y Y m M^{\mathrm{d}} F f, X X M^{\mathrm{d}} M^{\mathrm{d}} F f, X Y M^{\mathrm{d}} M^{\mathrm{d}} F f$ and $\left.Y Y M^{\mathrm{d}} M^{\mathrm{d}} F f\right)$ with frequencies $x_{1} \ldots x_{10}$, and eight

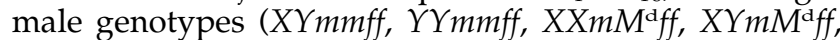
$Y Y m M^{\mathrm{d}} f f, X X M^{\mathrm{d}} M^{\mathrm{d}} f f, X Y M^{\mathrm{d}} M^{\mathrm{d}} f f$ and $\left.Y Y M^{\mathrm{d}} M^{\mathrm{d}} f f\right)$ with frequencies $y_{1} \ldots y_{8}$. Males homozygous for $M^{\mathrm{d}}$ have fertility $0 \leqslant u \leqslant 1$ and viability $0 \leqslant v_{m} \leqslant 1$, whereas females homozygous for $M^{d}$ have viability $0 \leqslant v_{f} \leqslant 1$. Again, a Maple 11 version of the system of 18 recursion equations is available for inspection upon request.
Invasion of $M^{\text {d }}$ into the standard $\mathrm{XY}$ system: The relevant $9 \times 9$ Jacobian matrix restricted to the subspace without the $F$ allele has leading eigenvalue $\lambda=2 k>1 \Leftrightarrow k>\frac{1}{2}$, hence a driving $M^{\mathrm{d}}$ allele can always invade, regardless of its effects on fertility and viability in homozygous condition. The new equilibrium, in which all males are heterozygous for $M^{\mathrm{d}}$ and $Y$ is lost, is stable $\left(\lambda_{1}=1 /(2 k)<1 \Leftrightarrow k>\frac{1}{2}\right)$.

Invasion of $F$ into the $X X / M^{\mathrm{d}} \mathrm{m} / f f$ system: The leading eigenvalue of the full $18 \times 18$ Jacobian matrix is again given by (10). Hence $\lambda_{1}$ is independent of $u$ and $v_{m}$, and increases with $v_{f}$. For full viability of homozygous females $\left(v_{f}=1\right), \quad \lambda_{1}>1 \Leftrightarrow k>\frac{1}{2}$, whereas for complete inviability $\left(v_{f}=0\right), \lambda_{1}>1 \Leftrightarrow k>\frac{3}{5}$. Thus, even for relatively weakly driving $M^{\mathrm{d}}$ alleles $\left(\frac{1}{2} \leqslant k \leqslant \frac{3}{5}\right), F$ can always invade provided viability of $M^{\mathrm{d}} M^{\mathrm{d}}$ females is sufficiently high.

Numerical iterations (Table 4) suggest that after invasion of $F$, for sufficiently high values of $k, Y$ can once again invade and go to fixation (for lethality $k \geqslant 0.8$, sterility $k>0.5$ ). Numerical calculations of eigenvalues confirm these invasion conditions. 